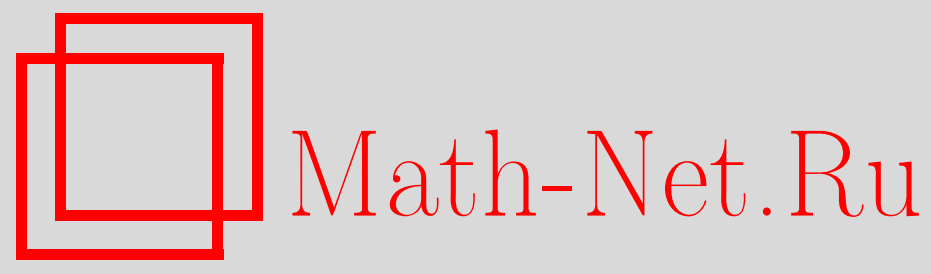

О. М. Шинкаренко, Е. С. Корчак, Создание динамической расчётной модели рабочего хода пресса с насосноаккумуляторным приводом, Вестн. Сам. гос. техн. ун-та. Сер. Физ.-мат. науки, 2012, выпуск 2(), 124131

DOI: https://doi.org/10.14498/vsgtu944

Использование Общероссийского математического портала Math-Net.Ru подразумевает, что вы прочитали и согласны с пользовательским соглашением

http: //www.mathnet.ru/rus/agreement

Параметры загрузки:

IP: 52.87 .193 .239

26 апреля 2023 г., 15:12:46

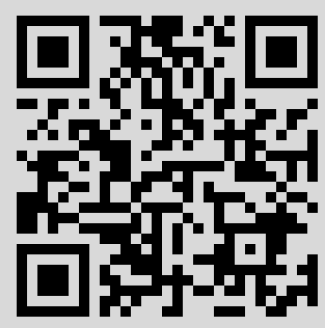


Вестн. Сам. гос. техн. ун-та. Сер. Физ.-мат. науки. 2012. № 2 (27). С. 124-131

УДК 517.958:621.226

\title{
СОЗДАНИЕ ДИНАМИЧЕСКОЙ РАСЧЁТНОЙ МОДЕЛИ РАБОЧЕГО ХОДА ПРЕССА С НАСОСНО-АККУМУЛЯТОРНЫМ ПРИВОДОМ
}

\author{
О. М. Шинкаренко, Е. С. Корчак
}

Донбасская государственная машиностроительная академия, 84313, Украина, Краматорск, ул. Шкадинова, 72.

E-mail: helen_korchak@ukr.net

\begin{abstract}
Рассмотрены особенности движения подвижной поперечины гидравлического пресса на различных этапах рабочего хода на базе типовой экспериментальной осииллограммы. Приведена расчётная схема рабочего хода с указанием принятых допущений «жёсткой» модели гидропривода. Разработаны динамические расчётные модели первого и второго этапов рабочего хода пресса с насосно-аккумуляторным приводом. Дано описание коэффициентов дифференииального уравнения Рикатти, приведено его аналитическое решение относителъно реалъного ковочного пресса. Подробно рассмотрены приведённые движущиеся массы металла и жидкости, вязкое гидравлическое сопротивление магистрали «аккумулятор - пресс» и активная сила пресса на рабочем ходу. Выявлено их влияние на движение поперечины на рабочем ходу. Проанализировано влияние конструктивной характеристики и времени закрытия регулирующего клапана на динамику торможения подвижной поперечины на рабочем ходу.
\end{abstract}

Ключевые слова: гидравлический пресс, динамическая модель, давление, подвижная поперечина, регулирующий клапан.

Введение. Рабочий ход является наиболее сложным и ответственным этапом машинного цикла пресса. В этот период осуществляется деформирование поковки до заданного размера. В рабочие цилиндры поступает жидкость высокого давления от насосно-аккумуляторной станции (НAC), а из возвратных цилиндров рабочая жидкость сливается в наполнительно-сливной бак (НСБ).

Исследования показали, что рабочий ход разделяется на несколько этапов, отличающихся друг от друга характером движения поперечины и действующими силами [1]. На рис. 1 показан пример осциллограммы, записанной на рабочем ходу пресса.

Из осциллограммы (рис. 1) видно, что подвижная поперечина, плавно разогнавшись после открытия впускного клапана $\left(h_{\mathrm{K}}\right)$, обжимает поковку с почти постоянной или несколько снижающейся скоростью. При закрытии клапана (кривая 1), поперечина (кривая 2) заметно притормаживается и после его закрытия останавливается. Путь торможения поперечины $S_{\text {т }}$ при закрытии клапана может составлять от $2 \div 3$ мм до $10 \div 15$ мм. Искусство кузнеца заключается в том, чтобы вычислить момент начала закрытия клапана (фактически $S_{\text {т }}$ ) и остановить поперечину на заданном размере в преде-

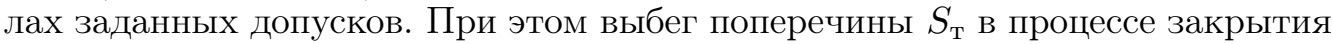
клапана зависит от целого ряда факторов: скорости поперечины, давления рабочей жидкости, температуры поковки, времени закрытия клапана, вида его расходной характеристики, динамических характеристик пресса.

Олег Михайлович Шинкаренко (к.т.н., доц.), доцент, кафедра машин и технологии обработки металлов давлением. Елена Сергеевна Корчак (к.т.н.), доцент, кафедра машин и технологии обработки металлов давлением. 
Если ковка ведётся в автоматическом режиме, в алгоритм системы автоматического управления должна быть заложена математическая модель рабочего хода и исходные параметры пресса. Таким образом, проблема создания адекватной математической модели рабочего хода гидравлического пресса является очень важной для производства.

Из рис. 1 можно заметить, что признаком, по которому рабочий ход пресса можно разделить на этапы, являются моменты срабатывания регулирующего клапана. По этому признаку рабочий ход можно разделить на три этапа: разгон поперечины на поковке при открывающемся клапане, движение при полностью открытом клапане и торможение поперечины при закрывающемся клапане. Для упрощения математической модели первый и второй этапы можно объединить в один: $T_{\mathrm{px}}=T_{\mathrm{p}+\mathrm{y}}+$ $+T_{\mathrm{T}}$

Построим расчётную схему рабочего хода пресса (рис. 2, а), на которой параметры имеют такие обозначения: $S_{\text {п }}$, $V_{\text {п }}$ - путь и скорость поперечины; $p_{\mathrm{a}}, p_{б}$, $p_{\mathrm{p}}, p_{\mathrm{B}}$ - давление в НАС, НСБ, рабочих и возвратных цилиндрах; $F_{\mathrm{p}}, F_{\text {в }}$ площади плунжеров рабочих и возвратных цилиндров; $F_{\text {тр }}$ - сила механического трения в узлах уплотнений плунжеров и направляющих поперечины; $\xi_{\mathrm{p}}$,

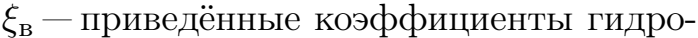
сопротивления магистралей «НАС - рабочий цилиндр» и «возвратные цилиндры - НСБ»; $L_{\mathrm{p}}, L_{\mathrm{B}}$ - приведённые дли-

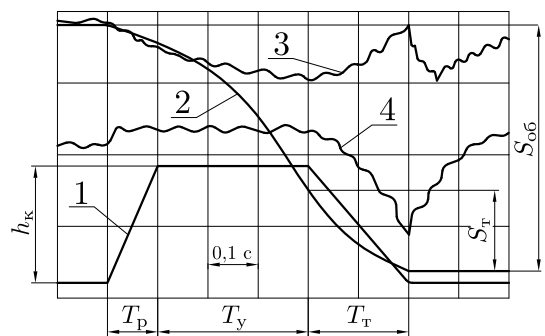

Рис. 1. Осциллограмма движения подвижной поперечины пресса на рабочем ходу: 1 - ход клапана; 2 - путь подвижной поперечины; 3 - давление жидкости после клапана (в рабочем цилиндре); 4 - давление перед клапаном (от НАC); $h_{\mathrm{K}}$ - текущая величина хода клапана; $S_{\mathrm{T}}$ - путь торможения; $T_{\mathrm{p}}, T_{\mathrm{y}}, T_{\mathrm{T}}$ - время разгона, установившегося движения и торможения; $S_{\text {об }}$ величина обжатия поковки

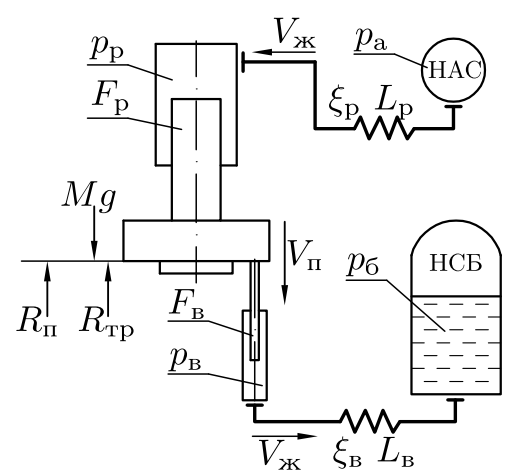

a

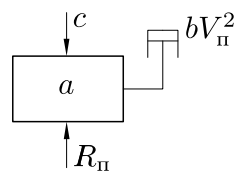

б

Рис. 2. Расчётная схема рабочего хода (а) и динамическая модель его первого этапа (б) ны магистралей; $M g$ - сила веса подвижных частей пресса; $R_{\Pi}$ - сила сопротивления поковки деформированию.

\section{1. Расчётная динамическая модель первого этапа рабочего хода пресса.} Первый этап рабочего хода начинается после открытия впускного клапана рабочих цилиндров. Подвижная поперечина, которая после холостого хода неподвижно лежала на поковке, разгоняется и деформирует её. Для математического описания динамики первого этапа рабочего хода пресса наиболее подходит «жёсткая» модель. Её основные допущения такие [2]:

- взаимодействия в системе распространяются мгновенно;

- жидкость имеет постоянную вязкость, несжимаема и нетеплопроводна;

- трубопроводы и элементы конструкции пресса абсолютно жёсткие; 
- гидравлические потери пропорциональны квадрату скорости жидкости;

- давление НАС и НСБ постоянно;

- впускной клапан рабочих цилиндров открывается мгновенно;

- сопротивление поковки деформированию постоянно.

Принятые допущения вполне адекватны для первого этапа рабочего хода и существенно не влияют на картину движения подвижной поперечины пресса на рабочем ходу, но значительно упрощают математический аппарат модели, позволяя решить её дифференциальные уравнения аналитически и получить алгебраические выражения, описывающие движения поперечины. В действительности давления в НАС и НСБ непостоянны и изменяются в пределах $\pm 5 \%$. Впускной клапан, как видно из рис. 1, открывается не мгновенно, а за 0,1 с. Сопротивление поковки несколько увеличивается к концу обжатия. Но, как показали исследования, эти изменения существенно не влияют на характер движения поперечины на первом этапе рабочего хода пресса.

Составим на основании расчётной модели (рис. 2, а) дифференциальное уравнение движения подвижной поперечины:

$$
M \frac{d V_{\Pi}}{d t}=p_{\mathrm{p}} F_{\mathrm{p}}+M g-p_{\mathrm{B}} F_{\mathrm{в}}-R_{\mathrm{тр}}-R_{\Pi} .
$$

Суммарная сила трения $R_{\text {тр }}$ складывается из сил трения в уплотнительных манжетах $\approx(0,03 \div 0,04) P_{\text {п }}$ и направляющих поперечины $\approx(0,05 \div 0,1) \mathrm{Mg}$. При этом $P_{\Pi}$ - номинальное усилие, развиваемое прессом.

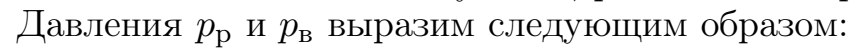

$$
p_{\mathrm{p}}=p_{\mathrm{a}}-0,5 \rho \xi_{\mathrm{p}} V_{\Pi}^{2}-\rho L_{\mathrm{p}} \frac{d V_{\Pi}}{d t}, \quad p_{\mathrm{B}}=p_{\text {б }}+0,5 \rho \xi_{\mathrm{B}} V_{\Pi}^{2}+\rho L_{\mathrm{B}} \frac{d V_{\Pi}}{d t},
$$

где $0,5 \rho \xi_{\mathrm{p}, ~ в} V_{\Pi}^{2}-$ потери на преодоление гидросопротивлений в соответствующих магистралях; $\rho L_{\mathrm{p}, \text { в }} d V_{\text {п }} / d t$ - потери на разгон столба жидкости в начале движения поперечины (потери на создание инерционного напора). Приведённые коэффициенты $\xi_{\mathrm{p}}$ и $\xi_{\text {в }}$ (гидросопротивления магистралей рабочих и возвратных цилиндров) можно определить по выражению

$$
\xi_{\mathrm{p}, \mathrm{\text {в }}}=F_{\mathrm{p}, \mathrm{\text {в }}}^{2}\left(\lambda \sum_{i=1}^{n} \frac{l_{i}}{d_{i} f_{i}^{2}}+\sum_{i=1}^{n} \frac{\zeta_{i}}{f_{i}^{2}}\right),
$$

где $\lambda \approx 0,3$ - коэффициент потерь на трение на прямых участках трубопроводов; $d_{i}, l_{i}$ - диаметры и длины участков трубопроводов; $f_{i}$ - площади проходного сечения участков трубопроводов; $\zeta_{i}$ - коэффициенты местных гидравлических сопротивлений на участках трубопроводов.

Приведённые длины трубопроводов определяются по выражению

$$
L_{\mathrm{p}, \text { в }}=F_{\mathrm{p}, \text { в }} \sum_{i=1}^{n} \frac{l_{i}}{d_{i}^{2}} .
$$

Подставляя (2) в исходное дифференциальное уравнение (1) и группируя члены, получим

$$
\begin{aligned}
\left(M+\rho L_{\mathrm{p}} F_{\mathrm{p}}+\rho L_{\mathrm{B}} F_{\mathrm{B}}\right) \frac{d V_{\Pi}}{d t}+0,5 \rho\left(\xi_{\mathrm{p}} F_{\mathrm{p}}+\xi_{\mathrm{B}} F_{\mathrm{B}}\right) V_{\Pi}^{2}- & \\
& -M g-p_{\mathrm{a}} F_{\mathrm{p}}+p_{б} F_{\mathrm{B}}+R_{\mathrm{Tp}}+R_{\Pi}=0 .
\end{aligned}
$$


Введём следующие обозначения:

$M+\rho\left(L_{\mathrm{p}} F_{\mathrm{p}}+L_{\mathrm{B}} F_{\mathrm{B}}\right)=a, \quad 0,5 \rho\left(\xi_{\mathrm{p}} F_{\mathrm{p}}+\xi_{\mathrm{B}} F_{\mathrm{B}}\right)=b, \quad M g+p_{\mathrm{a}} F_{\mathrm{p}}-p_{б} F_{\mathrm{B}}-R_{\text {тр }}=c$.

Запишем уравнение (3) с учётом принятых обозначений:

$$
a \frac{d V_{\Pi}}{d t}+b V_{\Pi}^{2}-c+R_{\Pi}=0
$$

Это известное уравнение Рикатти для первого этапа рабочего хода пресса, которое благодаря постоянству коэффициентов $a, b$ и $c$ решается аналитически [3]. Рассмотрим коэффициенты уравнения (4).

Коэффициент $a$-приведённые к площадям плунжеров рабочих и возвратных цилиндров движущиеся массы металла и жидкости в магистрали «НАC - пресс». При этом необходимо отметить, что приведённые массы жидкости на $2 \div 3$ порядка превосходят по величине массу движущихся металлических частей, и именно они определяют динамику всей машины. Чем длиннее магистраль «НАС - пресс» и чем меньше диаметр её проходного сечения, тем выше приведённые массы жидкости в системе, тем выше уровень гидравлических ударов на прессе и ниже его производительность. Необходимо так проектировать гидравлическую систему, чтобы аккумулятор находился рядом с прессом и соединялся с ним трубопроводом достаточно большого диаметра. Это резко снизит приведённые массы жидкости и, соответственно, динамическую составляющую работы пресса.

Коэффициент $b$ характеризует вязкое гидравлическое сопротивление движению поперечины. Чем длиннее магистраль «НАС - пресс», чем больше в ней установлено арматуры, чем меньше диаметр её проходного сечения, тем больше гидравлические потери в системе, тем ниже скорость подвижной поперечины и производительность пресса. Рекомендации по снижению коэффициента $b$ такие же, как и для коэффициента $a$. Кроме того, для снижения коэффициента $b$ магистраль «НАС - пресс» необходимо максимально разгрузить от излишней арматуры и других местных сопротивлений.

Коэффициент $c$ - активная сила пресса на рабочем ходу.

В уравнении (4) первый член $a\left(d V_{\text {п }} / d t\right)$ - инерционная сила Даламбера, условно прикладываемая к системе в периоды неустановившегося движения (разгона и торможения) поперечины. Второй член $b V_{\Pi}^{2}-$ сила вязкого гидравлического сопротивления движению поперечины.

На рис. 2, б приведена динамическая модель первого этапа рабочего хода. На подвижные массы $a$ действуют три силы: $c, R_{\text {п и }} b V_{\Pi}^{2}$.

Инерционная сила $a\left(d V_{\text {п }} / d t\right)$ на модели не указана, т.к. она в зависимости от режима движения поперечины меняет свой знак. Если превалируют активные силы, то идёт разгон поперечины и инерционная сила направлена против движения. Если же идёт торможение поперечины, т.е. преобладают силы сопротивления, то инерционная сила помогает движению и препятствует торможению. Уравнение (4) приводится к табличному интегралу, который вычисляется аналитически. Обозначим в уравнении (4) $c-R_{\Pi}=\tilde{c}$ и запишем

$$
a \frac{d V_{\Pi}}{d t}+b V_{\Pi}^{2}-\tilde{c}=0,
$$


то есть постоянное сопротивление поковки деформированию включили в свободный член уравнения (5). Разрешая (5), получим алгебраические выражения изменения пути, скорости и ускорения поперечины в функции времени $t$ после открытия впускного клапана рабочих цилиндров:

$$
S_{\Pi}=\frac{a}{b} \ln \left(\operatorname{ch}\left(\frac{\sqrt{\tilde{c} b}}{a} t\right)\right), \quad V_{\Pi}=\sqrt{\frac{\tilde{c}}{b}} \operatorname{th}\left(\frac{\sqrt{\tilde{c} b}}{a} t\right), \quad j_{\text {п }}=\frac{\tilde{c}}{a}\left(1-\operatorname{th}^{2}\left(\frac{\sqrt{\tilde{c} b}}{a} t\right)\right) .
$$

При $t \rightarrow \infty \operatorname{th}(t) \rightarrow 1$, т.е. скорость поперечины стремится к постоянной наибольшей установившейся величине, а ускорение - к нулю. Таким образом, значение наибольшей скорости составляет

$$
V_{\Pi_{\max }}=\sqrt{\tilde{c} / b}=\sqrt{\left(c-R_{\Pi}\right) / b}
$$

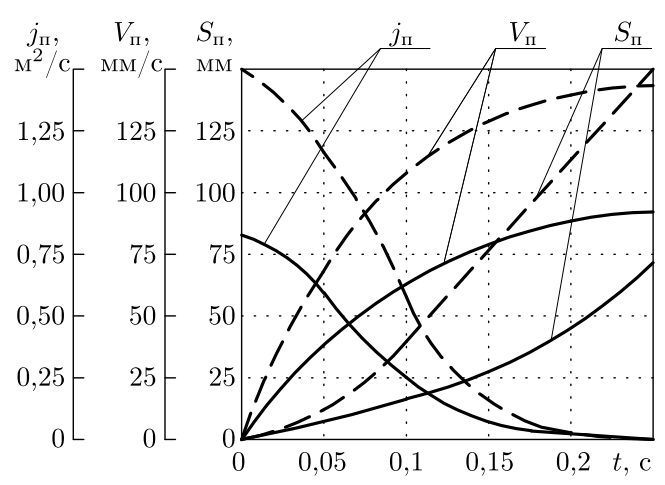

Рис. 3. Кривые разгона поперечины пресса при разных сопротивлениях поковки деформированию: сплошные линии $-R_{\Pi}=0,7 c$, штриховые линии $-R_{\text {п }}=0,1 c$

На рис. 3 приведены кривые (сплошные линии) изменения пути, скорости и ускорения подвижной поперечины ковочного пресса усилием 40 MН на первом этапе рабочего хода. Сила сопротивления поковки составляет $70 \%$ активной силы пресса $\left(R_{\Pi}=0,7 c\right)$. Для сравнения штрихом показаны кривые движения поперечины при $R_{\text {п }}=0,1 c$. Как видно из рассмотрения кривых, сила сопротивления поковки деформированию существенно влияет на параметры движения поперечины. С ростом сопротивления поковки скорость поперечины замедляется, а время разгона до установившейся скорости рас-

тёт [4].

2. Расчётная динамическая модель второго этапа рабочего хода. Второй этап рабочего хода является более ответственным, т.к. на этом участке формируется заданный размер поковки. Затормаживание поперечины необходимо обеспечить за минимальное время, без гидроударов и в пределах заданных допусков. Рассмотрим процесс торможения подвижной поперечины более подробно.

На втором этапе рабочего хода поперечина деформирует поковку при закрывающемся клапане. Из осциллограммы на рис. 1 мы видим, что в процессе закрытия клапана скорость поперечины существенно замедляется и после его закрытия она останавливается. Но фактически клапан не тормозит поперечину, т.к. он установлен на входе в рабочий цилиндр. Клапан отсекает от цилиндра источник жидкости высокого давления, разделяя магистраль «НАС пресс» на два участка: «НАС - клапан» и «клапан - рабочий цилиндр». Подвижная поперечина останавливается, когда гидростатическая сила рабочего цилиндра уравновешивается силой сопротивления поковки деформированию. Это подтверждается и осциллограммой на рис. 1. 
На первом участке «НАС - клапан» давление жидкости увеличивается по мере закрытия клапана, образуя пик в момент его закрытия. На втором участке наоборот - давление в процессе закрытия клапана понижается, образуя в момент закрытия клапана пик в сторону понижения. Расхождение давлений в разные стороны объясняется тем, что столб жидкости, двигавшийся от НАС к рабочим цилиндрам, разделился на две части, каждая из которых в момент разделения обладала определённым запасом кинетической энергии. У столба жидкости перед клапаном, для которого трубопровод оказался полностью перекрытым, запас кинетической энергии перешёл в энергию давления, что наблюдается на осциллограмме. Столб жидкости после клапана в силу своей инерционности продолжал перемещаться к рабочим цилиндрам, в связи с чем у клапана сформировалась волна пониженного давления. Чем несовершеннее расходная характеристика регулирующего клапана, чем ближе она к релейной, тем больше расхождение давлений перед и после клапаном и больше вероятность гидравлического удара в системе управления прессом. Таким образом, на втором этапе рабочего хода металлические подвижные части и жидкость, двигавшиеся на первом этапе как одна масса, приобретают характер двухмассовой системы. При этом разделителем масс служит закрывающийся регулирующий клапан.

Математическая модель из одномассовой «жёсткой» становится двухмассовой «жёстко-упругой». В качестве первой массы принимаем приведённую к площади плунжеров рабочих цилиндров массу жидкости на первом участке магистрали «НАС - клапан» (до клапана), а в качестве второй - сумму масс металла и приведённую массу жидкости на втором участке («клапан - рабочий цилиндр»). В качестве элемента, связывающего обе массы, принимаем невесомую пружину, параметры которой соответствуют приведённой упругости второго участка гидропривода. Поскольку второй период рабочего хода является продолжением первого, за основу при составлении его математической модели возьмём приведённое уравнение Рикатти (4), разделив его на два уравнения, связанных между собой упругой составляющей:

$$
\left\{\begin{array}{r}
a_{1} \frac{d^{2} S_{1}}{d t^{2}}+b\left[1+\alpha\left(1-\frac{t}{t_{3}}\right)^{-2 n}-\alpha\right]\left(\frac{d S_{1}}{d t}\right)^{2}-c_{1}-K_{1}\left(S_{2}-S_{1}\right)=0 \\
a_{2} \frac{d^{2} S_{2}}{d t^{2}}-c_{2}+K_{1}\left(S_{2}-S_{1}\right)+R_{\Pi}=0 .
\end{array}\right.
$$

Здесь $a_{1}, a_{2}$ - приведённые движущиеся массы на первом и втором участках гидропривода; $c_{1}, c_{2}$ - активные силы на участках гидропривода; $S_{1}, S_{2}-$ пути первой и второй приведённых масс; $\alpha$ - коэффициент качества гидросистемы; $t_{3}$ - время закрытия клапана при торможении поперечины; $n$ - показатель вида характеристики клапана; $K_{1}$ - приведённая жёсткость второго участка гидропривода.

Член в квадратных скобках первого уравнения системы (6) представляет собой безразмерный нелинейный коэффициент, характеризующий рост приведённого гидросопротивления регулирующего клапана при его закрытии. Коэффициент $\alpha$ указывает на степень искажения расходной характеристики клапана под влиянием общего гидравлического сопротивления магистрали «НАС - клапан». Система (6) аналитически не разрешается из-за наличия переменных коэффициентов при производных. Её решение возможно только 
в численном виде. На рис. 4 приведены расчётные кривые торможения первой и второй приведённых масс, полученные при закрытии регулирующего клапана за 0,01 с, 0,1 с и 0,2 с. Уравнения модели решались применительно к ковочному гидравлическому прессу усилием $63 \mathrm{MH}$ [5].
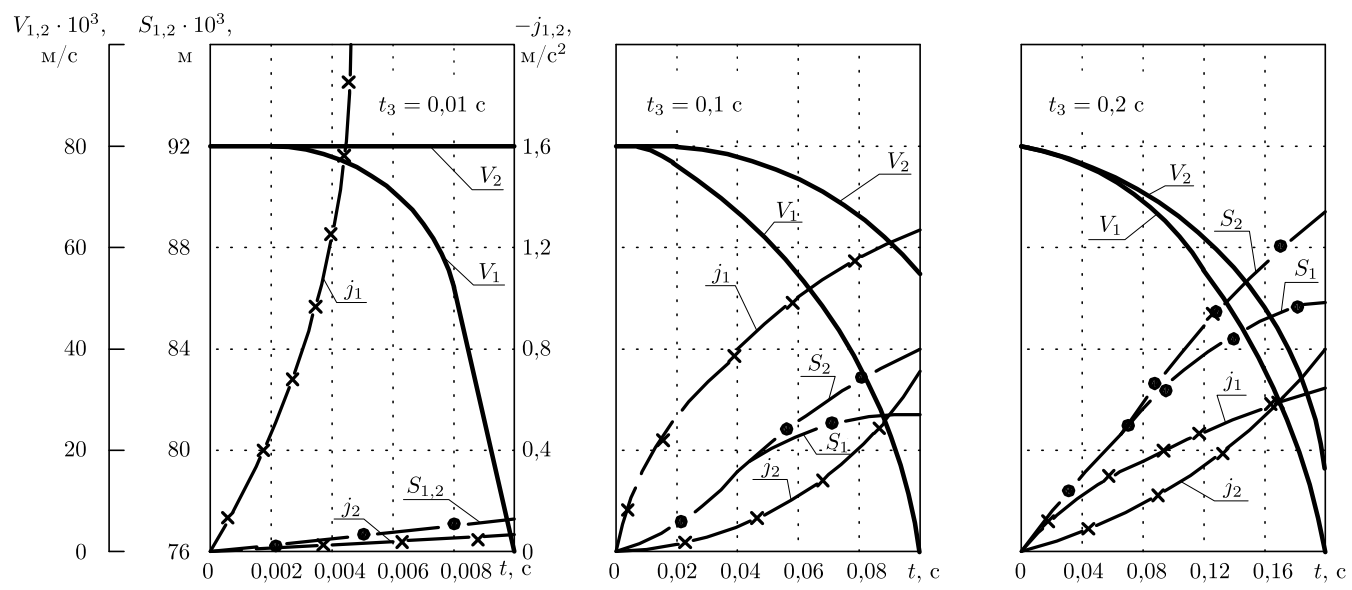

Рис. 4. Влияние времени закрытия клапана с линейной конструктивной характеристикой на динамику торможения подвижной поперечины пресса

Из рассмотрения кривых видим, что при очень малом времени закрытия $\left(t_{3}=0,01 \mathrm{c}\right)$ регулирующий клапан интенсивно тормозит массу жидкости на первом участке магистрали, но не успевает оказать заметного воздействия на поперечину, которая в момент закрытия клапана имеет почти ту же скорость, что и в начале закрытия $V_{2}=80 \mathrm{~mm} / \mathrm{c}$. Это обуславливает низкую точность ковки. Кроме того, ускорение $j_{1}$, с которым тормозится масса жидкости $a_{1}$, недопустимо велико $\left(j_{1}=36 \mathrm{~m} / \mathrm{c}^{2}\right)$, что говорит о наличии в магистрали интенсивного гидравлического удара. С увеличением $t_{3}$ регулирующий клапан оказывает все более заметное влияние на характер движения поперечины. Так, при $t_{3}=0,1$ с скорость поперечины $V_{2}$ снижается с 80 до $55 \mathrm{~mm} / \mathrm{c}$, а при $t_{3}=0,2 \mathrm{c}$ - до $12 \mathrm{~mm} / \mathrm{c}$. Ускорение первой массы $a_{1}$ в двух последних случаях во много раз меньше $\left(1,3\right.$ и $\left.0,8 \mathrm{~m} / \mathrm{c}^{2}\right)$, чем при $t_{3}=0,01$ с, т.е. гидроудара в системе в этих случаях не происходит.

Заключение. Таким образом, математическое моделирование позволяет подобрать оптимальные величины основных параметров рабочего хода пресса без построения физической модели или натурального образца машины. K таким параметрам относятся в первую очередь: время закрытия регулирующего клапана $t_{3}$, вид его конструктивной характеристики $n$, коэффициент качества гидросистемы $\alpha$.

\section{БИБЛИОГРАФИЧЕСКИЙ СПИСОК}

1. Соколов Л.Н., Золотухин В.Н., Ефимов В.Н. и др. Ковка слитков на прессах/ ред. Л. Н. Соколов. Киев: Техніка, 1984. 127 с. [Sokolov L. N., Zolotukhin V. N., Efimov V. N., et al. Forging Ingots in Presses / ed. L. N. Sokolov. Kiev: Tekhnika, 1984. 127 pp.]

2. Шинкаренко О. М., Корчак E. С. Совершенствование режимов работы гидравлических прессов с насосно-аккумуляторным приводом на холостом ходе / В сб.: Обработка ма- 
териалов давлением: Сб. научных трудов. Вып. 2 (27). Краматорск: ДГМА, 2011. С. 190194. [Shinkarenko O. M., Korchak E.S. Improved operating modes of hydraulic presses with a pump-accumulator drive at idle / In: Materials working by pressure: Collection of science papers. Issue 2 (27). Kramatorsk: DGMA, 2011. Pp. 190-194].

3. Белов А.Ф., Розанов Б. В., Лини, В. П. Объемная штамповка на гидравлических пресcax. М.: Машиностроение, 1986. 256 c. [Belov A. F., Rozanov B. V., Lints V. P. Bulk forming on hydraulic press. Moscow: Mashinostroenie, 1986. 256 pp.]

4. Золотухин Н. М., Шинкаренко О.М. Экспериментальное исследование сопротивления поковок деформированию при протяжке // Кузнечно-штамповочное производство, 1980. №3. C. 3-5. [Zolotukhin N. M., Shinkarenko O. M. Experimental study of forgings resistance to pulling deformation // Kuznechno-shtampovochnoe proizvodstvo, 1980. no. 3. Pp. 3-5].

5. Шинкаренко, О.М., Корчак, Е.С. Торможение подвижных частей ковочных прессов с насосно-аккумуляторным приводом // Извест. ТулГУ. Сер. Механика деформируемого твердого тела и обработка металлов давлением, 2006. №1. С. 346-355. [Shinkarenko O.M., Korchak E.S. Inhibition of moving parts of forging presses with pumpaccumulator drive // Izvest. TulGU. Ser. Mekhanika deformiruemogo tverdogo tela i obrabotka metallov davleniem, 2006. no. 1. Pp. 346-355].

Поступила в редакцию 17/IV/2011;

в окончательном варианте - 16/V/2012.

MSC: 93A30

\section{DESIGNING OF DYNAMIC CALCULATION MODEL OF PRESS WITH PUMP-ACCUMULATOR DRIVE WORKING STROKE}

\section{O. M. Shinkarenko, E.S. Korchak}

Donbass State Engineering Academy,

27, Shkadinov st, Kramatorsk, 84313, Ukraine.

E-mail: helen_korchak@ukr.net

Peculiarities of hydraulic press ram moving on different stages of the working stroke on the basis of experimental oscillogram are considered. Rated scheme of the working stroke with pointing out the assumptions of the "hard" hydraulic drive model is adduced. Dynamic calculation models of the first and second stages of the working stroke of hydraulic press with pump-accumulator drive are designed. Description of the coefficients of differential Ricatti equation is given, its analytical decision concerning real forging press is adduced. Presented moving metal-and-fluid masses, viscous hydraulic resistance of the line "accumulator - press" and the active press force on the working stroke are considered in detail. Its influence on ram moving on the working stroke is revealed. The influence of constructive characteristic and shutting time of the control valve on the dynamics of ram decelerating on the working stroke is analyzed.

Key words: hydraulic press, dynamic model, pressure, ram, control valve.

Original article submitted 17/IV/2011; revision submitted $16 / \mathrm{V} / 2012$.

Oleg M. Shinkarenko (Ph. D. (Tech.)), Associate Professor, Dept. of Machinery \& Metalforming Technology. Elena S. Korchak (Ph. D. (Tech.)), Associate Professor, Dept. of Machinery \& Metalforming Technology. 